\title{
Hollow cathode sustained plasma microjets: Characterization and application to diamond deposition
}

\author{
R. Mohan Sankaran and Konstantinos P. Giapis ${ }^{a)}$ \\ Division of Chemistry and Chemical Engineering, California Institute of Technology, Pasadena, \\ California 91125
}

(Received 11 April 2002; accepted for publication 10 June 2002)

Extending the principle of operation of hollow cathode microdischarges to a tube geometry has allowed the formation of stable, high-pressure plasma microjets in a variety of gases including Ar, $\mathrm{He}$, and $\mathrm{H}_{2}$. Direct current discharges are ignited between stainless steel capillary tubes ( $d$ $=178 \mu \mathrm{m}$ ) which are operated as the cathode and a metal grid or plate that serves as the anode. Argon plasma microjets can be sustained in ambient air with plasma voltages as low as $260 \mathrm{~V}$ for cathode-anode gaps of $0.5 \mathrm{~mm}$. At larger operating voltage, this gap can be extended up to several millimeters. Using a heated molybdenum substrate as the anode, plasma microjets in $\mathrm{CH}_{4} / \mathrm{H}_{2}$ mixtures have been used to deposit diamond crystals and polycrystalline films. Micro-Raman spectroscopy of these films shows mainly $s p^{3}$ carbon content with slight shifting of the diamond peak due to internal stresses. Optical emission spectroscopy of the discharges used in the diamond growth experiments confirms the presence of atomic hydrogen and $\mathrm{CH}$ radicals. (C) 2002 American Institute of Physics. [DOI: 10.1063/1.1497719]

\section{INTRODUCTION}

Microhollow cathode discharges or microdischarges are stable, high-pressure discharges formed in a cathode with a hole and an anode of arbitrary shape. ${ }^{1}$ It has been found experimentally that reducing the cathode hole diameter to near $100 \mu \mathrm{m}$ allows operation at atmospheric pressure in rare gases such as neon, ${ }^{2}$ argon, ${ }^{3}$ and xenon. ${ }^{3}$ In one version, a planar electrode geometry is used consisting of a layered structure ( $\sim 200 \mu \mathrm{m}$ thick) of two metal plates on either side of a dielectric spacer with a hole through the structure. ${ }^{4}$ Discharges are struck in the hole between the metal electrodes in direct current mode at voltages similar to those used for conventional low-pressure glow discharges but at much larger current density. The increased ionization is attributed to the Pendel effect ${ }^{5}$ which describes the oscillatory motion of electrons in the radial electric field created in the hollow cathode. Optical emission spectra from microdischarges formed in rare gases at high pressure contain lines from excimers ${ }^{3}$ and other highly excited states ${ }^{6}$ suggesting that high energy electrons are present. Efficient ionization at high pressure is desirable for materials processing and surface modification where these microdischarges could serve as sources of radicals and ions. We have recently reported one such application where $\mathrm{CF}_{4} / \mathrm{Ar}$ microdischarges were used to etch patterns in silicon. ${ }^{7}$

A preferred configuration for utilizing microdischarges as a radical or ion source is one in a flow geometry where species produced in the hollow cathode are transported to a substrate. In this arrangement, a tube is used as both the gas inlet and the cathode. In previous studies, such hollow cathode discharges were operated at lower pressures ( $p$ $<1$ Torr) ${ }^{8}$ consistent with the larger diameter tubes $(0.4-2$

\footnotetext{
a)Electronic mail: giapis@cheme.caltech.edu
}

$\mathrm{mm}$ ) employed; radio frequency power ${ }^{9,10}$ was also used which required complicated matching networks. Although atmospheric operation was achieved in one case, the discharges were found to form on the surface of the electrodes. ${ }^{11}$ Operation at high pressures in the hollow cathode mode requires shrinking of the hole diameter to sizes similar to those found in planar microdischarges such that the pressure times hole diameter $(p \cdot d)$ is on the order of 10 Torr $\mathrm{cm} .{ }^{1}$ Formation of microdischarges in a flow geometry would take advantage of the properties of a hollow cathode and could be attractive for thin film deposition. ${ }^{12}$ Moreover, the overall size encourages use in microelectromechanical systems applications where a miniaturized plasma source is desirable. ${ }^{13}$

In this article, we report on flow-stabilized microdischarges, termed "plasma microjets," using metal capillary tubes with hole sizes small enough to enable operation in the hollow cathode mode at high pressures. We focus on atmospheric pressure operation and characterize the dependence of the plasma microjet properties on flow rate and hole size. To demonstrate the usefulness of the plasma microjet as a source of growth species in thin film deposition, we also include results of diamond film deposition experiments using $\mathrm{CH}_{4} / \mathrm{H}_{2}$ gas mixtures.

\section{EXPERIMENTAL SETUP AND PROCEDURES}

Experiments to study the electrical and optical properties of the plasma microjet were done in Ar flowing into ambient air at atmospheric pressure. The setup for single and multiple tubes is schematically depicted in Fig. 1. Stainless steel capillary tubes (Varian, Inc.) with hole sizes $(d)$ ranging from 178 to $508 \mu \mathrm{m}$ and lengths of $5 \mathrm{~cm}$ were used as the cathode with a metal grid as the anode. The discharges were operated using a negative dc power supply $(0-5 \mathrm{kV})$ with a $25 \mathrm{k} \Omega$ 


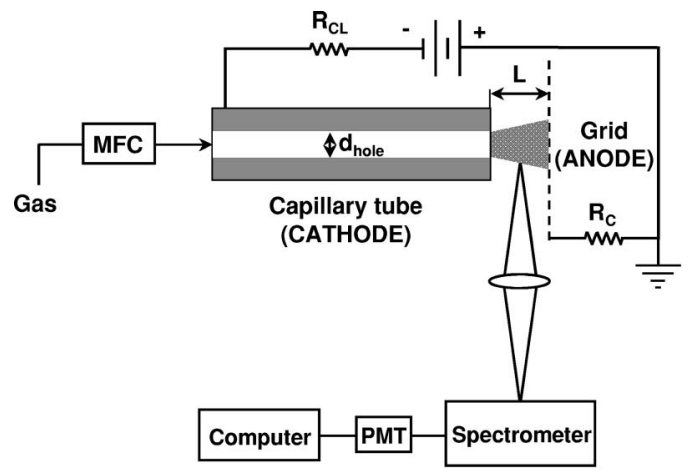

(a)

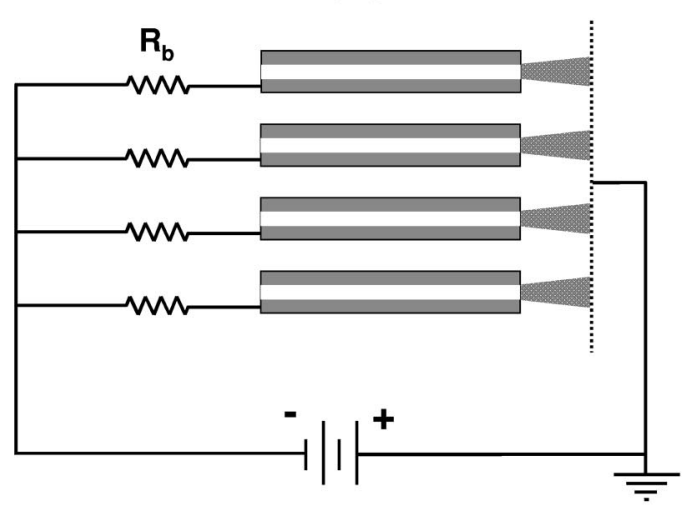

(b)

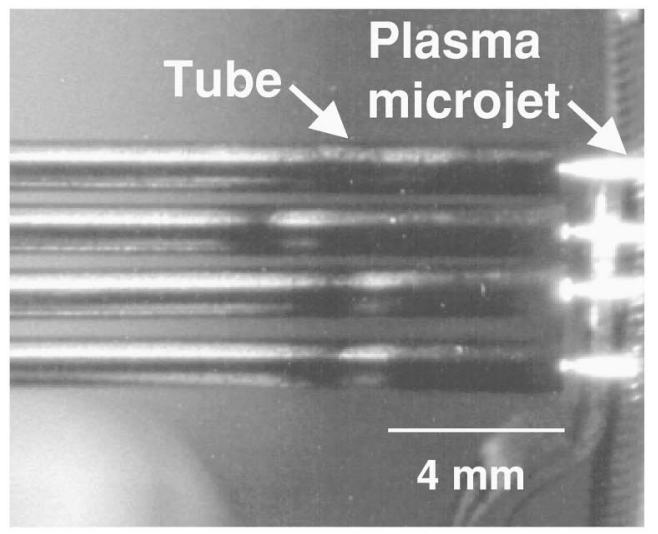

(c)

FIG. 1. (a) Schematic diagram of a single hollow cathode plasma microjet with optical emission spectroscopy setup. (b) Setup for parallel operation of four tubes using a ballast resistor for each tube but a single power supply. (c) A photo of four Ar plasma microjets operating in parallel at a cathode-anode gap of $2 \mathrm{~mm}$.

current-limiting resistor $\left(R_{\mathrm{CL}}\right)$ in series. The distance between the cathode and anode $(L)$ could be varied by moving either the tube or the grid using a micrometer. The plasma current was determined from the voltage drop across a $1 \mathrm{k} \Omega$ resistor in series $\left(R_{C}\right)$ and the plasma voltage was measured by a probe directly connected to the cathode. Gas flow through the tubes was adjusted using mass flow controllers. The discharge was characterized by optical emission spectroscopy. Spectra were acquired by using standard optics, a $0.22 \mathrm{~m}$ SPEX 1680 double monochromator (gratings ruled with 1200 grooves/mm, blazed at $630 \mathrm{~nm}$ ) and a Hamamatsu

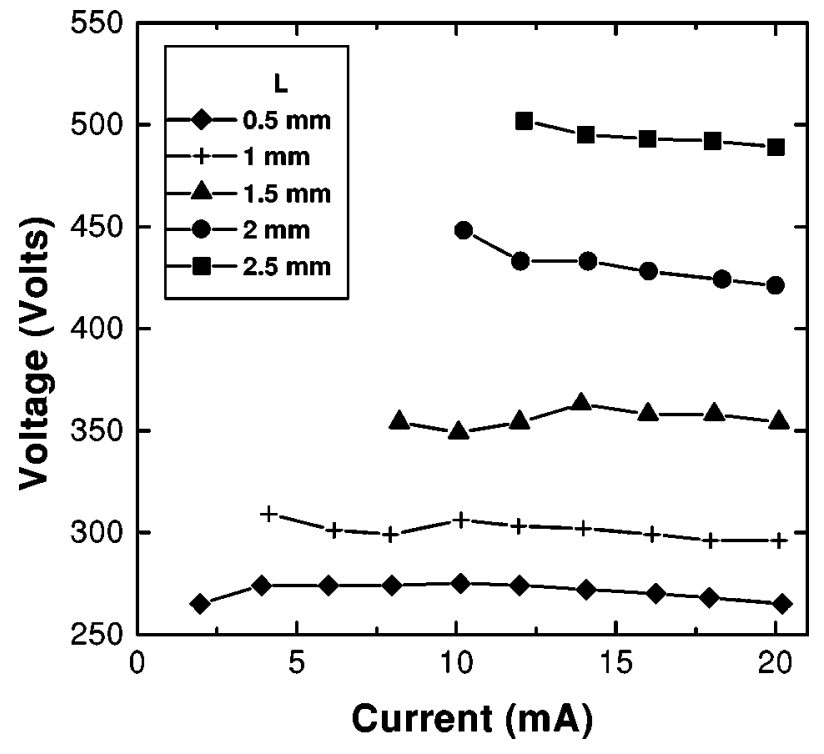

FIG. 2. $I-V$ characteristics of an Ar plasma microjet flowing in ambient air at 760 Torr at various cathode-anode gap separations $(L)$. Argon flow rate $=100 \mathrm{sccm}$, capillary tube inner diameter $=178 \mu \mathrm{m}$.

R928 photomultiplier tube. Light was collected from the side of the plasma microjet as depicted in Fig. 1(a) thereby sampling radiation from the outflow region.

Diamond growth experiments were performed at subatmospheric pressure with a setup similar to Fig. 1(a) except for using a heated solid substrate as the anode. The substrates were polycrystalline molybdenum foils ( $99.98 \%$ purity); they were prepared by etching in a liquid solution of $\mathrm{HF} / \mathrm{HNO}_{3} / \mathrm{HCl}$ and by sonicating in acetone prior to placement in the growth chamber. No scratching by diamond powder was necessary to initiate growth. Before each experiment, the growth chamber was evacuated to $10^{-4}$ Torr, then filled with a fixed composition $\mathrm{H}_{2} / \mathrm{CH}_{4}$ mixture to 200 Torr at a flow rate of $100 \mathrm{sccm}$. Substrates were heated resistively to $800{ }^{\circ} \mathrm{C}$ with the temperature being measured in situ using a Pt/Pt-Rh thermocouple. Since the gas flow cooled the substrate, it was important that the thermocouple was in contact with the substrate directly behind the impingement point. Discharges were struck with the capillary tube end $2 \mathrm{~mm}$ away from the substrate while maintaining a constant current and voltage during the growth. After growing for 2-4 h, films were characterized by scanning electron microscopy (SEM), energy-dispersive spectroscopy (EDS), and microRaman spectroscopy (using a Renishaw M1000 Raman Spectrometer system with a $514.5 \mathrm{~nm}$ Ar laser).

\section{RESULTS AND DISCUSSION}

\section{A. Characteristics of the hollow cathode plasma microjet source}

The characteristics of the plasma microjet were studied as a function of the following parameters: plasma current and voltage, cathode-anode distance, and gas flow rate. $I-V$ traces of an Ar plasma microjet flowing in ambient air are shown in Fig. 2 for various cathode-anode gaps. The data were obtained by inducing gas breakdown with the gap as 


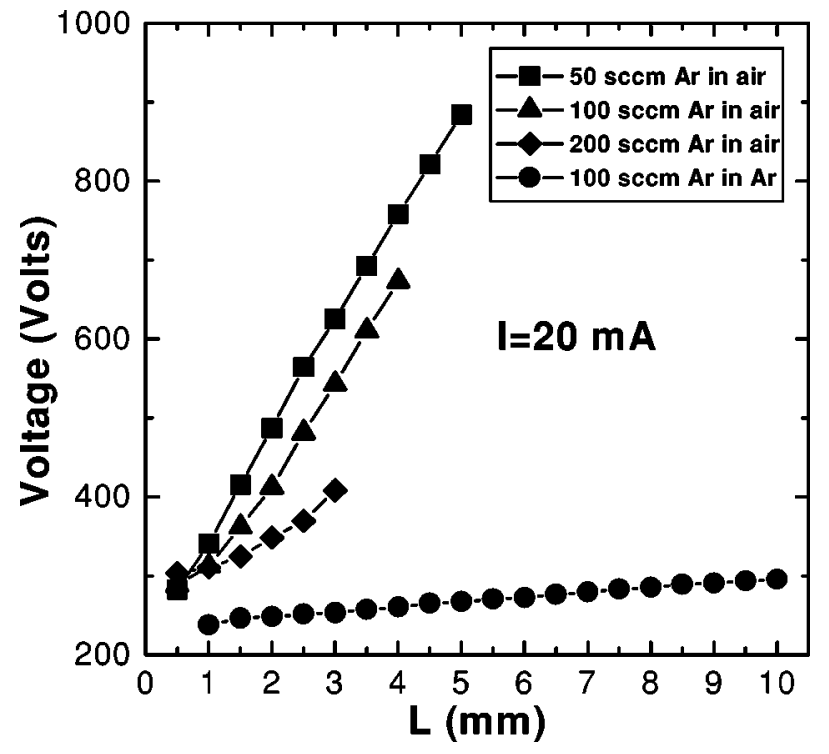

FIG. 3. Plasma voltage as a function of cathode-anode gap $(L)$ at various $\mathrm{Ar}$ flow rates for Ar plasma microjets flowing in ambient air or argon, as indicated. The flow rate is measured upstream from the capillary tube (inner diameter $=178 \mu \mathrm{m})$. The discharge current was fixed at $20 \mathrm{~mA}$.

small as possible $(<0.5 \mathrm{~mm})$, then moving the anode to extend the discharge. This procedure allowed the voltage required for gas breakdown to be less than $1 \mathrm{kV}$. The $I-V$ characteristics were reproducible and showed a weak voltage dependence over the current range selected. The slight drop in the plasma voltage as the current is increased may be due to heating of the electrodes which could cause thermionic emission of electrons. Indeed, the tube end begins to glow at high plasma currents, especially at low flow rates $(<100 \mathrm{sccm})$. At a gap of $0.5 \mathrm{~mm}$, the discharge is stable at currents and voltages as low as $2 \mathrm{~mA}$ and $260 \mathrm{~V}$, respectively. Sustaining the discharge at lower currents is possible if the gap is decreased further. When the gap is enlarged, the plasma voltage increases as shown more clearly in Fig. 3. The dependence is nonlinear at short gap distances, but becomes linear at larger gaps. This behavior suggests a constant electric field in the discharge which is reminiscent of static dc glow discharges where a linear dependence between voltage and interelectrode gap is observed over the length of the positive column at large cathode-anode separation. ${ }^{14}$ The minimum current required to prevent the discharge from extinguishing also changes significantly with gap separation. For example, while a current of $4 \mathrm{~mA}$ is adequate to sustain the discharge over $1 \mathrm{~mm}$, the current must be increased to 10 $\mathrm{mA}$ for a gap of $2 \mathrm{~mm}$. The discharge $I-V$ characteristics have important consequences for its application. Larger currents $(>20 \mathrm{~mA})$ and higher voltages $(>500 \mathrm{~V})$ are needed to obtain microjet lengths beyond $3 \mathrm{~mm}$.

Since the $I-V$ traces do not show any positive resistivity, parallel operation of plasma microjets using a single power source requires ballasting of individual discharges, as depicted in Fig. 1(b). The ballast resistor, $R_{b}$, must be identical in value to ensure that the current is the same in each discharge. This setup has been applied to parallel operation of four Ar plasma microjets, as shown in Fig. 1(c); the scheme can be easily expanded to larger arrays of tubes. When operating arrays of plasma microjets, the sustaining voltage was found to be the same as for a single microjet while the total current increased by a factor equal to the number of tubes. All plasma microjets in the array ignited simultaneously and each discharge behaved similarly to a single plasma microjet.

The current and voltage ranges required for Ar plasma microjet operation, especially at small gaps, are very similar to those used for planar Ar microdischarges. ${ }^{4}$ One major difference, however, is the flow which allows the discharge to be increased to lengths not possible with planar microdischarges. Another difference pertains to the absence of a dielectric material confining the discharge. Surface breakdown or deposits are believed to contribute to the reduction in lifetime of planar microdischarges. ${ }^{2}$ In contrast, plasma microjets can be run for hundreds of hours in rare gases without appreciable damage to the electrodes.

The influence of the flow rate on plasma voltage at various cathode-anode gaps is illustrated in Fig. 3. For these measurements, a gap distance is established, then the power supply voltage is adjusted to get a plasma current of $20 \mathrm{~mA}$, and finally the plasma voltage is recorded. A strong dependence of the plasma voltage on Ar flow rate was found for the experiments in ambient air at 760 Torr. The voltage required to sustain an Ar plasma microjet over a given gap size decreases significantly with the Ar flow rate. At a gap of 3 $\mathrm{mm}$, for instance, the voltage can be reduced from 625 to $408 \mathrm{~V}$ when the flow rate is increased from 50 to $200 \mathrm{sccm}$, respectively. The maximum distance that the Ar plasma microjet could be extended, however, decreased from 5 to 3 $\mathrm{mm}$ for the same flow rate change. Beyond the distances shown in Fig. 3 for each flow rate, the Ar plasma microjet could not be sustained. The behavior was significantly different from that observed for an Ar plasma microjet formed in ambient Ar at 760 Torr, also plotted in Fig. 3. In the latter case, the plasma voltage was much lower over the range of gap sizes tried and exhibited no dependence on the Ar flow rate. The microjet could also be extended over much larger gaps $(>10 \mathrm{~mm})$ as compared to the experiments in air.

From these observations, it appears that the effect of flow rate on plasma voltage may be related to the diffusion and mixing of air into the argon stream. A higher flow rate could reduce the mixing of air into the argon stream, particularly near the tube exit orifice. A reduction in the plasma voltage ensues then, as a consequence of the lower electric field requirement to sustain a discharge in argon with less air contamination. ${ }^{14}$ With increasing flow rate, however, the exit pressure and flow velocity become larger which could cause turbulence. Similar flow effects have been observed in $\mathrm{rf}$ discharges in high velocity gas flow where shock waves were observed. ${ }^{11}$ Turbulence could increase mixing with air at some distance from the exit of the tube ${ }^{15}$ thereby causing the discharge to extinguish at increased gaps. It should be noted that plasma microjets could not be formed when flowing air at atmospheric pressure using the $178 \mu \mathrm{m}$ diameter tube but operation at lower pressures $(<400$ Torr) was possible. Although mixing of ambient air into the argon stream could cause the observed flow rate dependence, heating effects and 

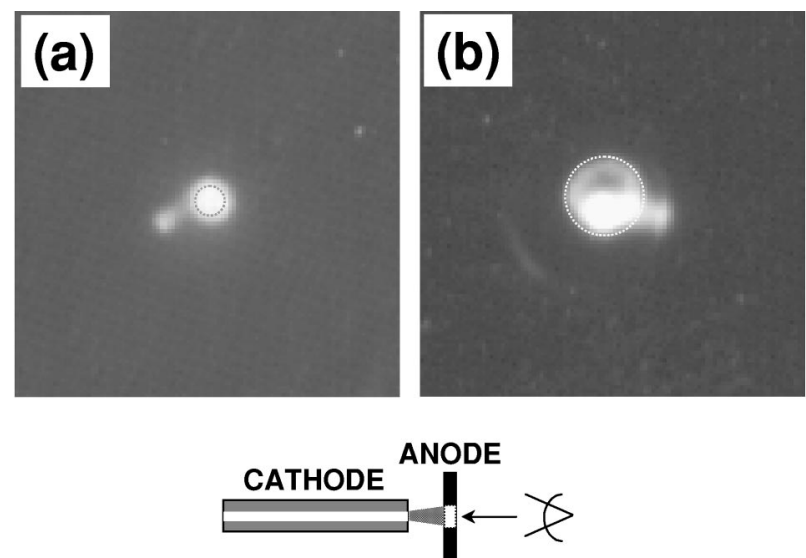

FIG. 4. Photographs of Ar plasma microjets in ambient air at 760 Torr in tubes with hole diameter of (a) 178 and (b) $508 \mu \mathrm{m}$. Photos were taken on axis through a metal aperture as depicted. The capillary tube hole boundary is outlined by a circle. Argon flow rate $=100 \mathrm{sccm}$, plasma current $=5 \mathrm{~mA}$.

plasma instabilities may also contribute to the observed complex behavior.

The discharges formed in tubes with hole diameters of $178 \mu \mathrm{m}$ are expected to operate in the hollow cathode mode at atmospheric pressure since their $p \cdot d$ value $(13.5$ Torr $\mathrm{cm}$ ) is close to an upper limit of 10 Torr $\mathrm{cm}$ experimentally found to be necessary for planar microdischarges. ${ }^{1}$ Figure 4 compares the appearance of an Ar plasma microjet formed in 178 and $508 \mu \mathrm{m}$ diameter tubes as seen through a concentric metal aperture $1 \mathrm{~mm}$ in diameter which serves as the anode. In the smaller diameter tube [Fig. 4(a)], the plasma forms in the center of the hole and stretches from the hole to the edge of the anode. The discharge appears to form outside the boundary of the hole (shown by the dotted line) but this may be caused by plasma expansion outside the hole and/or overexposure. This picture should be contrasted with the discharge in the larger inner diameter tube $(508 \mu \mathrm{m})$ shown in Fig. 4(b). The discharge does not fill the hole uniformly but appears to be localized near the inner surface of the tube. These observations are consistent with previous work on microdischarges ${ }^{4}$ where increasing the pressure for a given hole size caused a shift in appearance from a columnar to a ring-shaped plasma. In our experiment, we increase the tube inner diameter thereby changing the $p \cdot d$ term to a value that no longer supports operation in the hollow cathode mode.

The enhanced ionization expected from a hollow cathode mode of operation is corroborated by comparing emission spectra of Ar plasma microjets formed in the two different hole sizes (Fig. 5). Although most of the spectrum is composed of Ar neutral lines (415-435 nm) because of the high pressure, weaker intensity Ar ion lines appear in the spectrum from the plasma microjet formed in the smaller inner diameter tube $(178 \mu \mathrm{m})$. For the larger hole size of 508 $\mu \mathrm{m}$, these lines are absent from the spectrum consistent with weaker ionization. Lines corresponding to the second positive system of $\mathrm{N}_{2}$ also appear at 449.0, 457.4, and $466.7 \mathrm{~nm}$ indicative of mixing with air. Thus, it is necessary to shrink the hole size to the correct size regime in order to take ad-

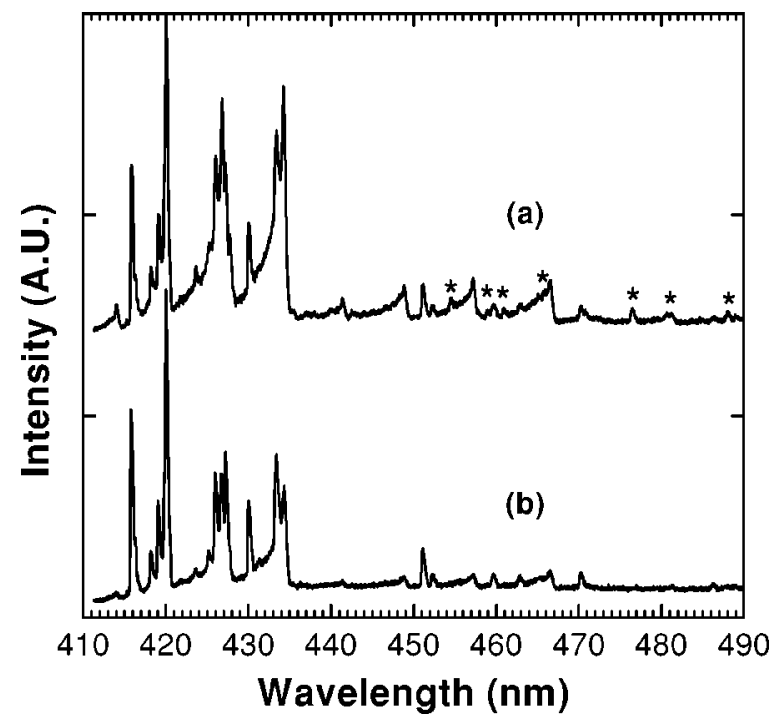

FIG. 5. Emission spectra of Ar plasma microjets in ambient air at 760 Torr in tubes with hole diameter of (a) 178 and (b) $508 \mu \mathrm{m}$. Argon ion lines marked by asterisk. Cathode-anode gap $=2 \mathrm{~mm}$, flow rate $=200 \mathrm{sccm}$, plasma current $=10 \mathrm{~mA}$.

vantage of the properties of a hollow cathode mode of excitation.

\section{B. Growth of diamond films using a $\mathrm{H}_{2} / \mathrm{CH}_{4}$ plasma microjet}

Plasma microjets could be utilized as convenient microreactors for the production of reactive radicals for etching, thin film deposition, or surface modification. As an example, we have used plasma microjets in $\mathrm{CH}_{4} / \mathrm{H}_{2}$ at pressures between 100 and 500 Torr to grow diamond films. For the pressures studied, the discharge was remarkably stable over a wide range of currents $(5-20 \mathrm{~mA})$ and could be run for hundreds of hours with very little damage to the tube. Representative emission spectra of $\mathrm{H}_{2}$ and $\mathrm{CH}_{4} / \mathrm{H}_{2}$ plasma microjets of variable composition are shown in Fig. 6. In pure $\mathrm{H}_{2}$, the strongest emissions are from atomic hydrogen Balmer lines at 486.1 and $656.3 \mathrm{~nm}$, noted as $\mathrm{H}_{\beta}$ and $\mathrm{H}_{\alpha}$, respectively. Many of the other lines in the spectrum are weaker $\mathrm{H}$ atom lines and $\mathrm{H}_{2}$ excited lines. When $\mathrm{CH}_{4}$ is added, lines appear near $430 \mathrm{~nm}$ corresponding to excited bands of the $\mathrm{CH}$ system. These lines become more intense with increasing $\mathrm{CH}_{4}$ concentrations. No lines corresponding to $\mathrm{C}_{2}$ or $\mathrm{CH}^{+}$were observed.

A single plasma microjet was used to grow diamond films employing gas mixtures consisting of $\mathrm{H}_{2}$ and varying amounts of $\mathrm{CH}_{4}$. The gases were mixed in a region upstream of the microjet using a constant $\mathrm{H}_{2}$ flow rate of $100 \mathrm{sccm}$ and $\mathrm{CH}_{4}$ flow rate between 0.1 and $1 \mathrm{sccm}$. In the following discussion, the percentage of $\mathrm{CH}_{4}$ is approximated by its flow rate. For discharge currents of $20 \mathrm{~mA}$, experiments could be run for several hours with the same tube; EDS of films did not detect any contamination from the electrode due to sputtering or evaporation. SEM images showed that the growth rate and morphology of the deposited films depend strongly on the $\mathrm{CH}_{4}$ concentration with significant changes below $1 \%$. At $0.5 \% \mathrm{CH}_{4}$, a somewhat continuous 


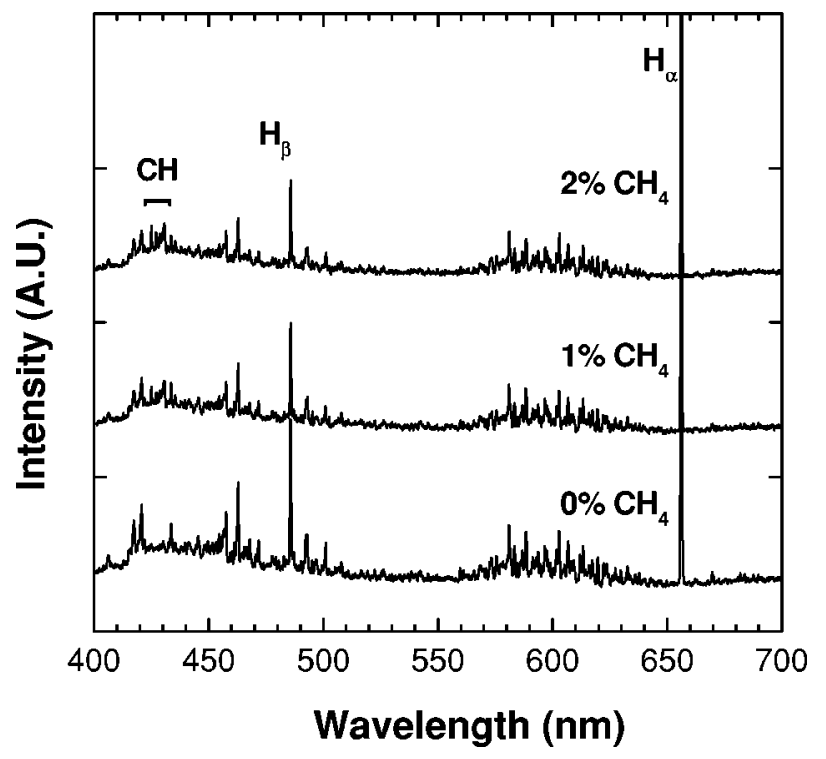

FIG. 6. Emission spectra of $\mathrm{H}_{2}$ and $\mathrm{CH}_{4} / \mathrm{H}_{2}$ plasma microjets at 200 Torr collected from the side of the discharge. Cathode-anode gap $=2 \mathrm{~mm}$, flow rate $=100 \mathrm{sccm}$, plasma current $=20 \mathrm{~mA}$.

film was deposited over a $500 \mu \mathrm{m}$ diameter area. The film consisted of dense particles near the center of the deposition area; the particle density decreased with distance away from the center. A close-up of the film [Fig. 7(a)] showed microscale roughness and some triangular faceting representative of diamond. As the $\mathrm{CH}_{4}$ concentration was reduced further to $0.25 \%$ and $0.1 \%$, individual crystals were observed with well-defined facets as shown in Figs. 7(b) and 7(c), respectively. Due to a drop in the growth rate, the films exhibited sparser coverage with the heaviest concentration of particles at the stagnation point. This type of isolated particle growth has been reported for an atmospheric-pressure inductively coupled plasma torch in a similar flow geometry. ${ }^{16}$ Interestingly, in that study there was little or no growth along the stagnation line, which was attributed to lower concentrations of atomic hydrogen at the center than near the edges of the substrate. The improved coverage at the center of the deposition area in our experiments is consistent with the highest concentration of excited states expected along the centerline of the hollow cathode.

Micro-Raman spectrocopy was performed to distinguish diamond-like carbon $\left(s p^{3}\right)$ from amorphous carbon $\left(s p^{2}\right)$ for each of the samples in Fig. 7 with the spectra shown in Fig. 8. The sharp peak that appears at approximately $1336 \mathrm{~cm}^{-1}$ is close to that of the first order optical phonon mode of natural diamond which occurs at $1332.5 \mathrm{~cm}^{-1}$. The shift in the peak is attributed to compressive stresses caused by lattice mismatch to a possible underlying $\mathrm{Mo}_{2} \mathrm{C}$ layer. ${ }^{16}$ Broad peaks at 1350 and $1580 \mathrm{~cm}^{-1}$, attributed to $s p^{2}$ carbon, were also present for films grown at the higher $\mathrm{CH}_{4}$ concentrations of $0.25 \%$ and $0.5 \%$. In the latter case, a shoulder at approximately $1150 \mathrm{~cm}^{-1}$ is also discernible which has been attributed to nanocrystals. ${ }^{17}$ This observation is consistent with the smaller grain size seen in the SEM image of this film [Fig. 7(a)]. As the $\mathrm{CH}_{4}$ concentration is reduced, the nondiamond peaks disappear and only a single peak at
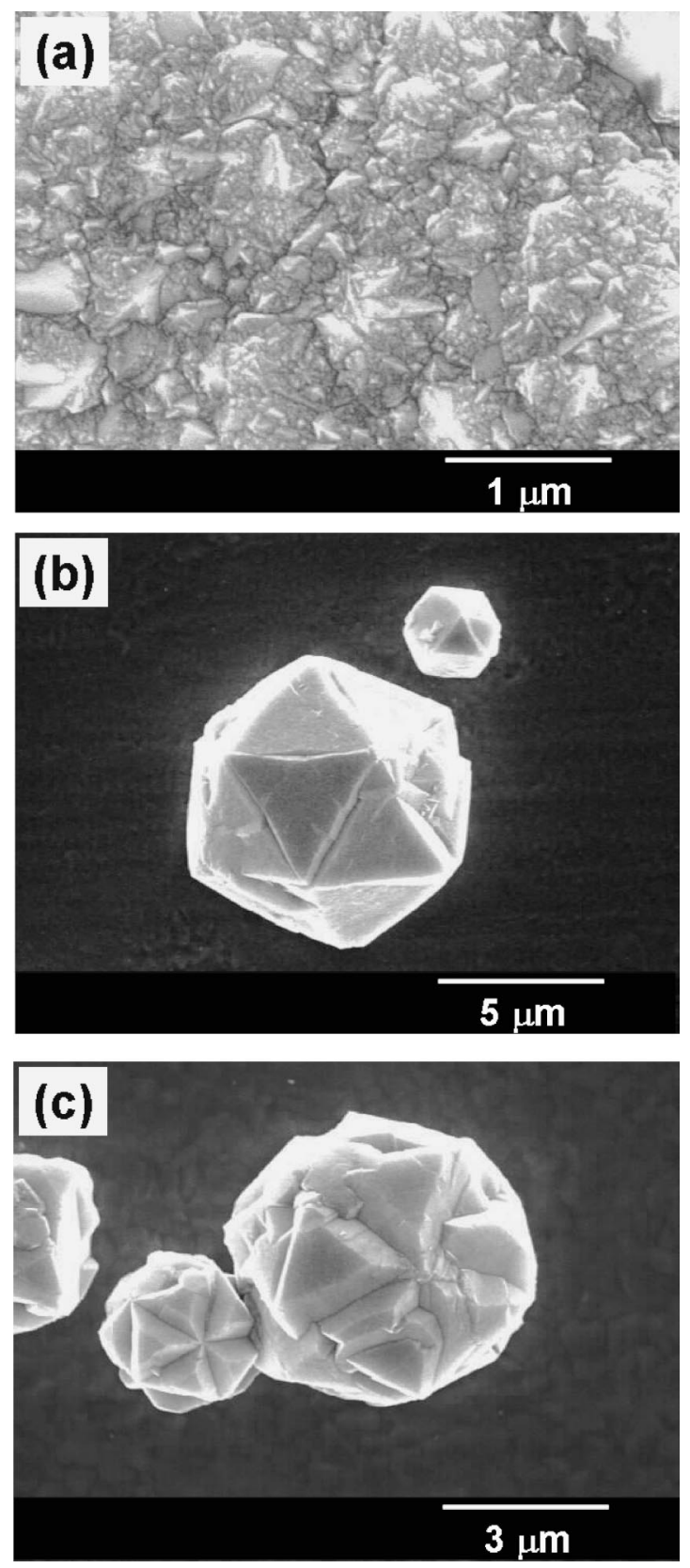

FIG. 7. SEM images of a diamond film and crystals grown using $\mathrm{CH}_{4} / \mathrm{H}_{2}$ plasma microjets at 200 Torr on molybdenum for $2 \mathrm{~h}$ at $\mathrm{CH}_{4}$ concentrations of (a) $0.5 \%$, (b) $0.25 \%$, (c) $0.1 \%$. Cathode-anode gap $=2 \mathrm{~mm}$, flow rate $=100 \mathrm{sccm}$, plasma current $=20 \mathrm{~mA}$, substrate temperature $=800{ }^{\circ} \mathrm{C}$.

$1336 \mathrm{~cm}^{-1}$ remains [Fig. 8(c)] suggesting a high purity microcrystalline diamond phase.

The diamond growth experiments demonstrate that the plasma microjet can be used as a reactive source for the deposition of films of comparable quality to those produced by other more complicated deposition techniques. The advantage of this tool is the simplicity of construction and operation, as well as the low power consumption. Thicker films are also possible since the source is stable and allows deposition of films continuously for much longer times. The coatings are restricted to small areas, but this may be desirable for materials processing on the microscale. Further, expanding to large arrays, plasma microjets could be utilized for the 


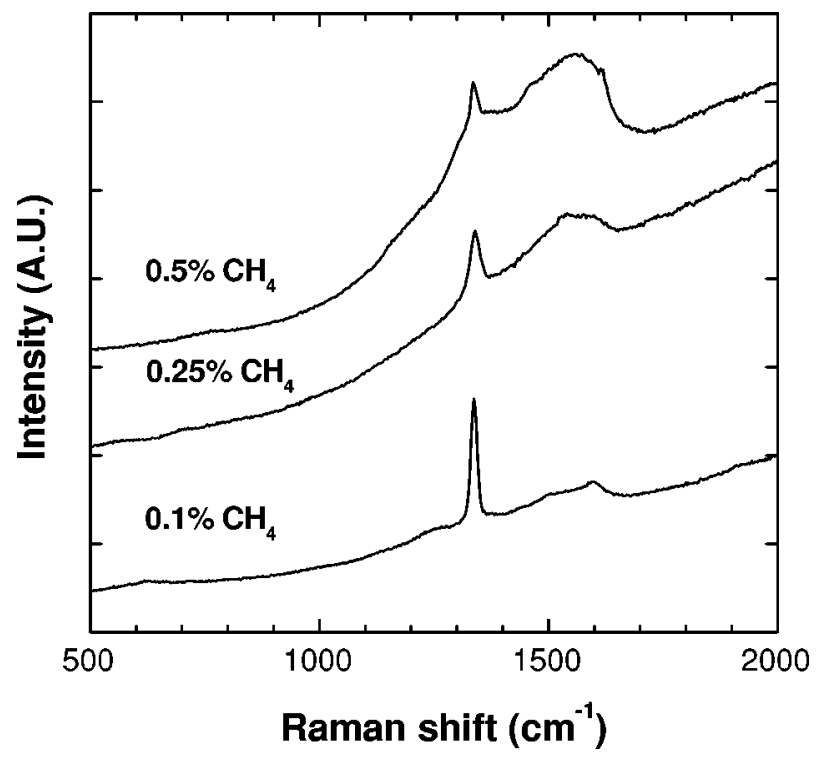

FIG. 8. Micro-Raman spectra of the diamond film and crystals corresponding to the micrographs shown in Fig. 7; growth conditions as stated in the caption of Fig. 7.

development of materials libraries for rapid screening and optimization purposes.

\section{SUMMARY}

The principle of operation of hollow cathode microdischarges has been extended to a tube geometry enabling in the formation of flow-stabilized plasma microjets. The microjets are capable of operating at high pressures, including 760 Torr, continuously for hundreds of hours with no discernible deterioration in performance. Optical emission spectroscopy of Ar plasma microjets in ambient air revealed emission from Ar ions which suggests intense ionization. The plasma microjets are very simple to construct, requiring a capillary tube with an appropriate inner diameter, an anode plate/grid of arbitrary shape, a gas flow through the tube, and a source of high voltage $(<1000 \mathrm{~V})$ dc power. The flow of gas associated with the formation of the microjet and the ability to operate stably at very low power makes the plasma microjets particularly useful as sources of radicals and ions for lab-ona-chip and microreactor applications. The ability to form arrays of plasma microjets using only one power supply make them attractive for efficient materials discovery where materials libraries could be developed to optimize rapidly plasma parameters, gas composition, and substrate deposition conditions. Plasma microjets in $\mathrm{CH}_{4} / \mathrm{H}_{2}$ have been used to optimize gas composition for growth of high quality diamond crystals. The low cost and simplicity of operation at 760 Torr also render plasma microjet arrays suitable for effluent processing for reduced environmental impact.

\section{ACKNOWLEDGMENTS}

The authors are grateful to Elizabeth Arredondo for technical assistance with the micro-Raman spectra. Useful discussions with R. C. Flagan are also acknowledged. This material was based on work supported by NSF (ECS-9729968).

${ }^{1}$ K. H. Schoenbach, R. Vergappen, T. Tessnow, and F. E. Peterkin, Appl. Phys. Lett. 68, 13 (1996).

${ }^{2}$ S.-J. Park, C. J. Wagner, C. M. Herring, and J. G. Eden, Appl. Phys. Lett. 77, 199 (2000).

${ }^{3}$ A. El-Habachi and K. H. Schoenbach, Appl. Phys. Lett. 72, 22 (1998).

${ }^{4}$ K. H. Schoenbach, A. El-Habachi, W. Shi, and M. Ciocca, Plasma Sources Sci. Technol. 6, 468 (1997).

${ }^{5}$ A. Guenterhschulze, Z. Phys. 19, 313 (1923).

${ }^{6}$ J. W. Frame, D. J. Wheeler, T. A. DeTemple, and J. G. Eden, Appl. Phys. Lett. 71, 1165 (1997).

${ }^{7}$ R. M. Sankaran and K. P. Giapis, Appl. Phys. Lett. 79, 593 (2001).

${ }^{8}$ L. Bardos, S. Berg, and H.-O. Blom, Appl. Phys. Lett. 55, 1615 (1989).

${ }^{9}$ L. Bardos and H. Barankova, Surf. Coat. Technol. 133-134, 522 (2000).

${ }^{10}$ A. Brablec, V. Kapicka, Z. Ondracek, P. Slavicek, M. Strecha, F. Stastny, and R. Vaculik, Czech. J. Phys. 49, 329 (1999).

${ }^{11}$ V. Kapicka et al., Plasma Sources Sci. Technol. 8, 15 (1999).

${ }^{12}$ F. Jansen, D. Kuhman, and C. Taber, J. Vac. Sci. Technol. A 7, 3176 (1989).

${ }^{13}$ J. A. Hopwood, J. Microelectromech. Syst. 9, 309 (2000).

${ }^{14}$ Y. P. Raizer, Gas Discharge Physics, 2nd ed. (Springer, Berlin, 1991).

${ }^{15}$ S.-I. Pai, Fluid Dynamics of Jets (Van Nostrand, New York, 1954).

${ }^{16}$ M. A. Cappelli, T. G. Owano, and C. H. Kruger, J. Mater. Res. 5, 2326 (1990).

${ }^{17}$ R. J. Nemanich, J. T. Glass, G. Lucovcky, and R. E. Shroder, J. Vac. Sci. Technol. A 6, 1783 (1988). 